\title{
Repositório Digital: instrumento de aprendizagem na Educação Profissional
}

\author{
Vinicius da S. Crizel ${ }^{1}$, Mônica U. Nunes ${ }^{1}$, Victor B. Coch $^{1}$, Juliana G. Vieira ${ }^{1}$, Liziane \\ G. Torchelsen ${ }^{1}$, Igor A. Pereira ${ }^{1}$, Viviani R. Kwecko ${ }^{1}$ \\ ${ }^{1}$ Observatório Regional do Trabalho e da Educação Profissional e Tecnológica - Instituto \\ Federal do Rio Grande do Sul (IFRS) - Campus Rio Grande \\ CEP - 96.201-460 - Rio Grande - RS - Brazil \\ vinicius.crizel845@gmail.com, monicaununes@gmail.com, \\ victorbarroscoch@gmail.com, juguerravieira@gmail.com, \\ liziane.garcia@riogrande.ifrs.edu.br, igor.pereira@riogrande.ifrs.edu.br, \\ viviani.kwecko@riogrande.ifrs.edu.br
}

\begin{abstract}
This work seeks to present the implementation and development of a digital repository and the simultaneous creation of it's website. Conceived by a group of computing students from the integrated technical course, this learning instrument for Vocational and Technological Education was divided in two steps: the development of the database and the implementation of the website, both using the programming languages SQL and PHP respectively. This platform aims to structure a repository for documents and reference data that reflect contextualized and updated information about the regional dimentions of Vocational and Technological Education (EPT) to serve as support for later studies.
\end{abstract}

Resumo. Este trabalho tem o objetivo de apresentar o desenvolvimento $e$ implementação de um repositório digital e a simultânea criação de um website. Concebido por alunos do curso Técnico em Informática para Internet integrado ao ensino médio, esse instrumento de aprendizagem para a Educação Profissional foi dividido em duas etapas: o desenvolvimento do banco de dados e a implementação do website, ambos utilizando linguagens de programação como $S Q L$ e PHP, respectivamente. A plataforma visa estruturar um centro de documentação de referência que reflita informações atualizadas $e$ contextualizadas sobre as dimensões regionais da Educação Profissional $e$ Tecnológica (EPT) para servir de apoio para estudos posteriores.

\section{Introdução}

Parte integrante de um projeto de desenvolvimento nacional, a Educação Profissional e Tecnológica está sendo convocada a formar jovens que transcendam as demandas técnicas do mundo do trabalho, articulando-as com a perspectiva de formação para o conhecimento reflexivo, crítico e relacional. Os sistemas de ensino e as escolas encontram-se frente a novas demandas formativas, transversalizadas principalmente pelas tecnologias de informação e comunicação - TICs. Estudos recentes sobre os processos do pensar e do aprender (LIBÂNEO, 2004) na contemporaneidade insistem que, além da acentuação do papel dos sujeitos na aprendizagem, a educação tem como necessidade desenvolver habilidades de pensamento e competências cognitivas, as quais objetivam romper com uma formação puramente técnica para criar espaços de diálogos entre Educação, Ciência e Trabalho. Nesse sentido, buscamos discutir os desafios da formação Técnica integrada em Informática para Internet, a partir da implementação de um Observatório Regional do 
Trabalho, Educação Profissional e Tecnológica. Esse lugar de onde falamos é importante para a compreensão do trabalho que desenvolvemos, ou seja, um processo de iniciação científica direcionado ao trabalhador e ao estudante do Ensino Médio.

O aproveitamento dos recursos oferecidos pela tecnologia, como por exemplo, o computador e a internet, depende de quem os usa. Para Masetto (2000) é importante não nos esquecermos de que a tecnologia possui um valor relativo, já que ela somente terá importância se for adequada para facilitar o alcance dos objetivos e se for eficiente para tanto. Há uma visão errada em torno do que é uma formação técnica em informática para internet e principalmente para o que ela serve. Frequentemente limitamos a atuação desse profissional a manutenção de computador, eliminação de vírus e/ou atualização de softwares. Entretanto, um técnico em informática para Internet está habilitado: a desenvolver softwares que facilitem a realização de tarefas ou resolução de problemas; a construir soluções que auxiliam o processo de criação de interfaces para intranet e internet; criar aplicativos empregados no comércio e marketing eletrônicos. Sendo assim, poderíamos afirmar que a formação do técnico em informática para internet oportuniza-o a resolver problemas de forma lógica utilizando, geralmente, um computador como ferramenta.

Um dos campos de maior atuação do técnico encontra-se associado ao desenvolvimento e manutenção de bancos de dados. Um banco é um sistema de organização de dados brutos a fim de gerar informações lógicas e providas de sentido. Funciona por meio de tabelas interligadas que interagem entre si, e que acionadas por comandos específicos, geram para seu usuário a informação desejada. Além de possuir a vantagem do tempo de processamento de grande volume de dados a partir de um ambiente virtual. Atualmente, outro setor que tem despertado interesse dos profissionais em informática se refere ao desenvolvimento de websites. Um website é um conjunto de páginas web cuja principal finalidade é conceder acesso rápido a um grande volume de informações e serviços disponibilizados por outros usuários.

O presente trabalho tem objetivo de apresentar o processo de implementação de um repositório digital a partir do desenvolvimento de seu banco de dados e a simultânea criação de seu website. Concebido por um grupo de alunos do curso Técnico em Informática para Internet na modalidade integrado ao ensino médio, como ferramenta de aprendizagem para a Educação Profissional e Tecnológica. Os repositórios institucionais, conforme definição do Instituto Brasileiro de Informação em Ciência e Tecnologia - IBICT (2012) são sistemas de informação que armazenam, preservam, divulgam e dão acesso à produção intelectual de comunidades universitárias. Contribuem para o aumento da visibilidade e o valor público das instituições, servindo como indicador tangível de sua qualidade; permitem a reforma do sistema de comunicação científica, expandindo o acesso aos resultados da investigação e reassumindo o controle acadêmico sobre a publicação científica (NETTO et al., 2012).

Este artigo está organizado da seguinte maneira: Na Seção 2 apresenta-se a metodologia adotada. A Seção 3 apresenta a discussão dos resultados obtidos notadamente o diagrama do sistema de banco de dados e do website. Finalmente, na Seção 4 apresentamse as conclusões e se discutem perspectivas de trabalhos futuros. 


\section{Caminhos Metodológicos}

O desenvolvimento do repositório digital iniciou em março de 2015, tendo sua previsão de término para dezembro de 2016. Representa uma nova etapa de um mapeamento acerca da qualificação do trabalhador. As respectivas fases que o antecederam representam coletas de dados, responsáveis por fornecer as informações a serem armazenadas e organizadas. $\mathrm{O}$ presente estudo envolve 4 discentes ligados ao curso de Informática para Internet do Instituto Federal de Educação, Ciência e Tecnologia - Campus Rio Grande.

$\mathrm{Na}$ primeira etapa desta fase do projeto foi desenvolvido o banco de dados para o qual utilizamos a linguagem de programação Structured Query Language ${ }^{l}$ (SQL) para sua criação e a linguagem de programação $P H P^{2}$ para a parte lógica. Para o armazenamento de conteúdo no banco de dados, optamos utilizar os conceitos do Sistema de Gerenciamento de Banco de Dados PostgreSQL ${ }^{3}$ (RIBAMAR, 2006), aproveitando a praticidade, agilidade e ferramentas disponibilizadas pelo mesmo. Na sequência foi esboçada a parte gráfica com o uso das linguagens $\mathrm{HTML}^{4}, \mathrm{CSS}^{5}$ e JavaScript ${ }^{6}$, além de softwares de manipulação de imagens como o Gimp ${ }^{7}$. Para a organização do projeto, backup e principalmente para a sincronização do trabalho dos integrantes, utilizamos o GitHub ${ }^{8}$ (BELL \& BEER, 2015), software de controle de versão que disponibiliza os repositórios para os arquivos. Essa tecnologia de controle de versão é essencial para a eficiência do trabalho em grupo. Atualmente o projeto encontra-se na parte de implementação do banco e hospedagem do site.

\section{Resultados e Discussão}

\subsection{Observatório Regional: o banco}

O Sistema de Gerenciamento de Banco de Dados (SGBD) utilizado é o PostgreSQL, banco de dados relacional que permite o armazenamento e indexação das informações obtidas por meio de entrevistas com trabalhadores, empregadores e docentes. O processo de desenvolvimento ocorre em diversas etapas: inicialmente com um protótipo do banco de dados, movendo-se para a implementação das interfaces. Na etapa de projeto foram criados diagramas relacionais e de entidade-relacionamento para o banco de dados. Na Figura 1 está exposto parte do diagrama relacional do banco, este que representa sua estrutura interna. Como exemplo, temos a tabela "trabalhadoresrespostas", que se relaciona com "perguntas", "trabalhadores" e "palavraschaves". Isso representa, de forma diagramada, uma estrutura básica de armazenamento e processamento de perguntas e respostas, sendo incluído nesse processamento uma separação de palavras-chave. Há também uma tabela chamada "stopwords", esta possui a função de armazenar termos considerados de pouca importância para a coleta de dados, como artigos e pronomes, sendo utilizados para a filtragem de palavras antes destas serem registradas.

\footnotetext{
${ }^{1}$ Disponível em <http://www.sqlcourse.com/intro.html>

${ }^{2}$ Disponível em <http://php.net/>

${ }^{3}$ Disponível em <https://www.postgresql.org/>

${ }^{4}$ Disponível em <http://www.w3schools.com/html/>

${ }^{5}$ Disponível em <http://www.w3schools.com/css/>

${ }_{7}^{6}$ Disponível em <http://www.w3schools.com/js/>

${ }^{7}$ Disponível em <https://www.gimp.org/>

${ }^{8}$ Disponível em <https://github.com/>
} 
V Congresso Brasileiro de Informática na Educação (CBIE 2016)

Anais do XXII Workshop de Informática na Escola (WIE 2016)

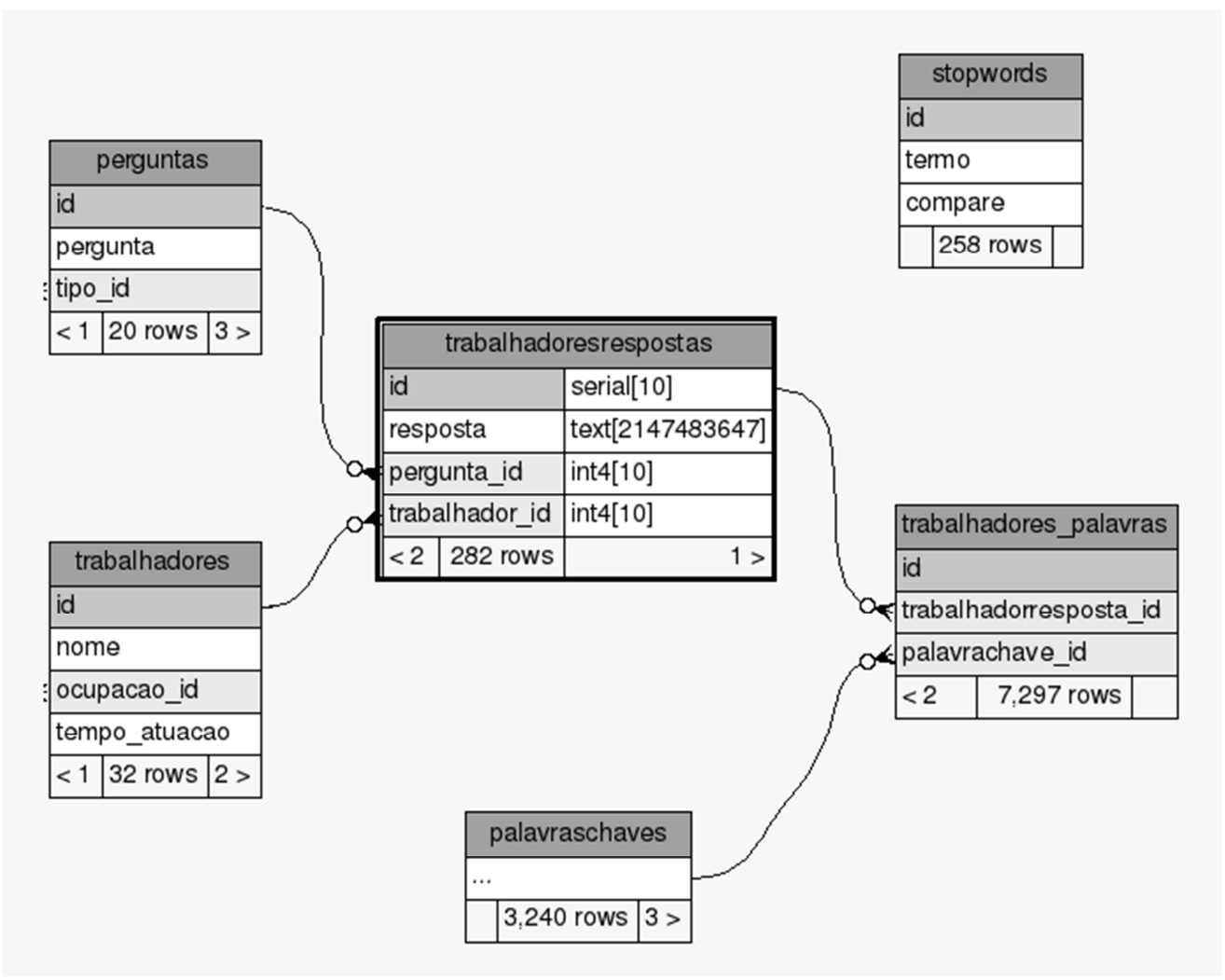

Figura 1 - Diagrama relacional

O sistema foi desenvolvido com objetivo de armazenar e organizar informações coletadas em um projeto cuja meta era mapear os saberes, informações e etapas da qualificação do trabalhador. As informações foram concebidas entre 2014 e 2015 e consideraram os discursos dos trabalhadores, docentes e empresários de parte do Arranjo Produtivo do Polo Naval e Energia (APL). Esse projeto realizou uma série de entrevistas com docentes e trabalhadores do polo naval, buscando entender e analisar de forma qualitativa e quantitativa, os aspectos referentes ao (i) perfil dos trabalhadores da cadeia de construção naval e offshore, (ii) a contratação e formação do quadro de funcionários e (iii) os benefícios concedidos. Associada a essa etapa foram investigados dados sobre a educação brasileira organizados pelos principais institutos de pesquisa. Dentre as fontes estão artigos científicos, relatórios de acompanhamento, apresentações e estudos realizados por instituições relevantes (ÍNDICE DE DESENVOLVIMENTO HUMANO MUNICIPAL BRASILEIRO, 2013; APL POLO NAVAL E OFFSHORE DE RIO GRANDE E ENTORNO, 2014; IBGE, 2014; APL POLO NAVAL E OFFSHORE/RS, 2015).

A percepção dos trabalhadores inseridos no APL, com relação a qualificação profissional, também foi investigada através de entrevistas conduzidas a partir da metodologia do Discurso do Sujeito Coletivo (DSC), com adaptações (LEFEVRE \& LEFEVRE, 2005; LEFEVRE \& LEFEVRE, 2010). Essas entrevistas foram realizadas na área industrial de uma das empresas inseridas no APL e conduzidas seguindo 4 áreas temáticas: (i) pré-requisitos na formação e qualificação profissional para atuar na área, (ii) processo de admissão na empresa e a relação desses com a formação na área de atuação; (iii) programas de formação continuada ofertados pela empresa ou fomentos oferecidos para a capacitação profissional; (iv) relação entre a qualificação profissional e o desempenho do trabalhador; (v) relação entre a falta de qualificação profissional e os 
índices de acidente de trabalho. Essas entrevistas foram então transcritas e inseridas no repositório, para serem armazenadas, organizadas e indexadas.

Os empresários foram investigados a partir de entrevistas realizadas na $3^{\text {a }}$ Feira do Polo Naval - RS, durante a Rodada de Negócios promovida pelo Serviço Brasileiro de Apoio às Micro e Pequenas Empresas no Rio Grande do Sul (SEBRAE-RS). Nessa oportunidade, foram realizadas 38 entrevistas, contemplando empresas ligadas, majoritariamente, ao setor metal-mecânico, prestadoras de serviços e automação industrial. O questionário foi composto por 19 perguntas divididas em 2 grandes eixos: (i) perfil do entrevistado e (ii) contratação e formação do quadro de trabalhadores.

\subsection{Observatório Regional: o website}

O sistema web desenvolvido para o projeto permitirá a representação dos dados em tabelas paginadas com filtros, gráficos e modelos estatísticos, de modo a tornar o conteúdo de fácil acesso e interpretação. Essa plataforma esta sendo construída com o CakePHP ${ }^{9}$, framework que utiliza a programação Orientada a Objetos (OO) aplicada a linguagem $P H P$. Durante a implementação do sistema, é usado o GitHub, software de controle de versão que mantém o desenvolvimento do sistema, pois disponibiliza repositórios que facilitam o backup e sincronização do trabalho dos integrantes. Esse sistema permitirá a criação de vários modos de apresentação e processamento de dados. $\mathrm{O}$ ambiente de desenvolvimento integrado (IDE) usado é a ferramenta NetBeansIDE ${ }^{10}$ pois possui boa integração com o framework CakePHP. Essa opção permite o uso de ferramentas de depuração, para rápida identificação e correção de erros, que junto ao navegador Mozilla Firefox ${ }^{11}$, que permite avaliarmos as funcionalidades e aparência do site.

O CakePHP é um framework de desenvolvimento rápido para $P H P$, livre e de código aberto. Foi escrito com o objetivo de oferecer uma estrutura que possibilite aos programadores desenvolverem aplicações robustas. Nossa opção pelo uso dessa ferramenta foi devida ao sistema $M V C(D E A C O N, 2013)$, que facilita a manutenção do projeto. O MVC (Model-View-Controller) se trata de um padrão de projeto (design pattern) e possibilita desenvolvimento rápido a partir de pacotes modulares. Essa aplicação possui 3 partes principais: Model, View e Controller. Para explicar seu funcionamento, primeiramente devemos entender o Controlador (Controller), que contém a lógica do programa. Ele funciona interpretando os comandos do usuário e consultando o Modelo (Model), responsável pela manipulação e validação de dados, para realizar operações. A visão (View) não sabe o que está sendo processado, apenas é responsável pela interação com o usuário, em forma de componentes gráficos e textos.

\footnotetext{
${ }^{9}$ Disponível em <http://cakephp.org/>

${ }^{10}$ Disponível em <https://netbeans.org/>

11 Disponível em <https://www.mozilla.org/pt-BR/firefox/new/>
} 
V Congresso Brasileiro de Informática na Educação (CBIE 2016)

Anais do XXII Workshop de Informática na Escola (WIE 2016)

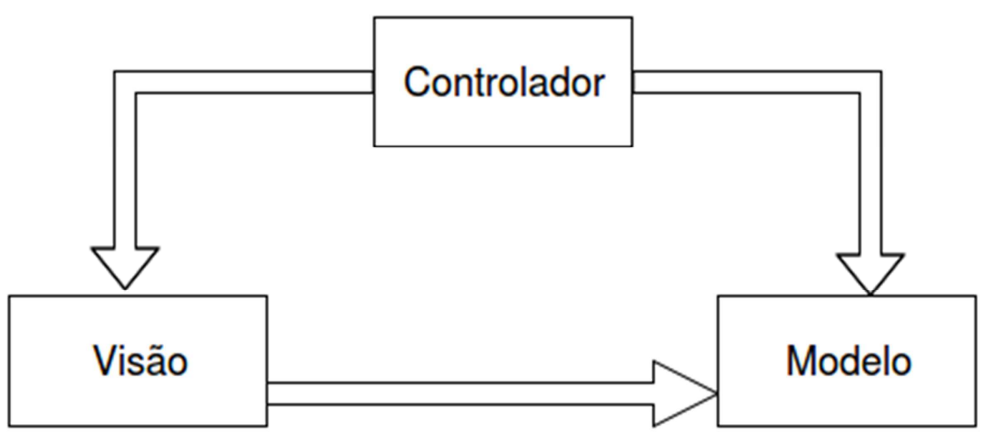

Figura 2 - Requisição no $M V C$

Segundo CARVALHO \& SILVA \& SIMÕES (2005), para desenvolver um website o programador deve questionar-se acerca do fácil acesso do usuário a informação, e se essa possui qualidade e credibilidade. Procuramos, durante o desenvolvimento do website, considerar tais observações através da organização lógica de distribuição do conteúdo por trás da criação dos menus e submenus, demonstrando a possibilidade do usuário de navegar até seu destino em, no máximo, 3 cliques. Outro elemento refere-se a origem da informação que, nesse repositório, está vinculada a outras pesquisas sobre o tema aplicadas pelo instituto e por outros repositórios de referência. Para o desenvolvimento do site, utilizou-se o diagrama da Figura 3 como base para organização das informações. É importante observar que o website encontra-se em fase de transformação do conteúdo estático para dinâmico, porém seu layout está pronto.

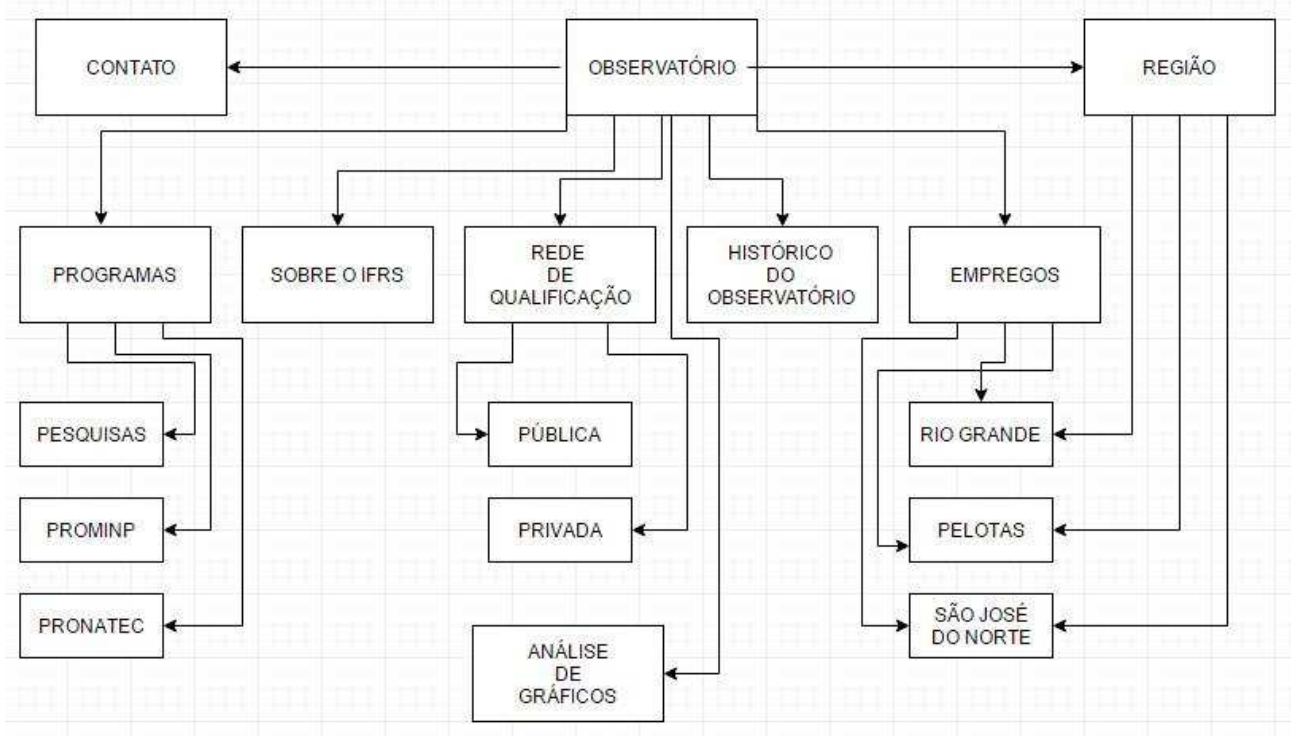

Figura 3 - Diagrama de construção do site

O website foi estruturado de maneira que possua na página inicial apenas cinco menus, como expostos na Figura 3, o número reduzido se deve ao objetivo de facilitar a busca pelo seu conteúdo.

A página inicial, chamada de index.html, foi elaborada para facilitar a navegação no website, que é constituída de quatro partes como mostra a Figura 4: (i) Cabeçalho: refere-se 
V Congresso Brasileiro de Informática na Educação (CBIE 2016)

Anais do XXII Workshop de Informática na Escola (WIE 2016)

à parte superior que contém a logomarca do Observatório construída no Gimp e cinco botões de menu; (ii) Seção 1: apresenta informações regionais das cidades que compõem o Arranjo Produtivo Local - APL; (iii) Seção 2: informa o histórico do Observatório Regional do Trabalho, Educação Profissional e Tecnológica; e por fim as (iv) Instituições Parceiras que representam organizações de compartilhamento dos dados que são armazenados e organizados neste repositório. As Figuras 4 e 5 apresentam essa criação visual do site. Possuindo uma interface agradável, o layout permite que o usuário se sinta confortável durante o acesso, para que possa consultar as informações geradas através dos dados contidos no banco de dados. Na Figura 6 destacamos a identidade visual desenvolvida para o site.

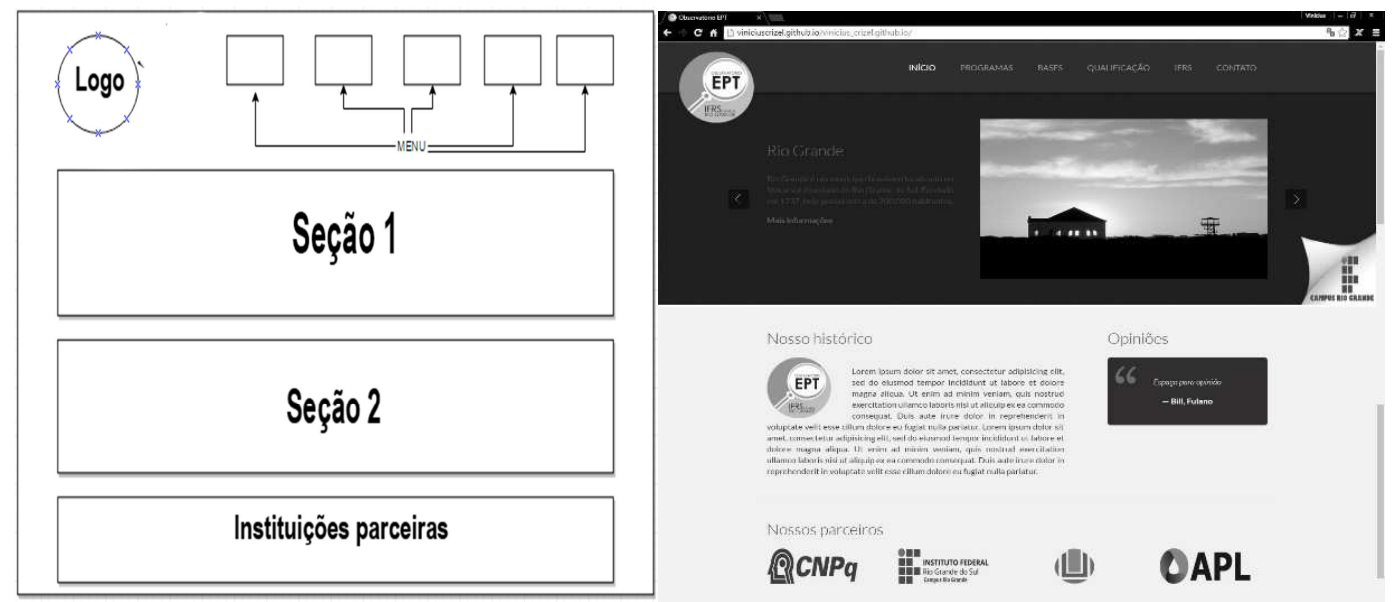

Figura 4 - Esboço do Website

Figura 5 - Página inicial do Website

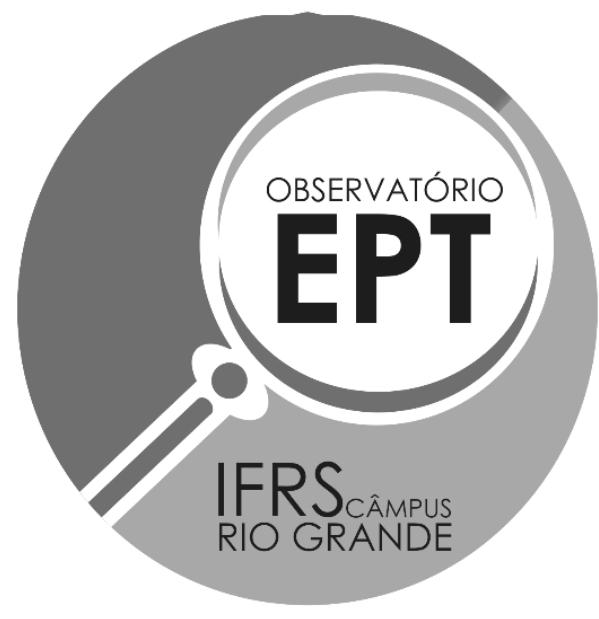

Figura 6 - Logomarca do Observatório

A partir desta seção inicial, o usuário tem acesso a todo o website. Em todas as páginas, é disponibilizado o endereço da instituição mantenedora e o e-mail para contato com a equipe do Observatório. Em relação ao conteúdo das informações disponibilizadas no website, destaca-se a preocupação em manter a clareza e objetividade facilitando, desta forma, o entendimento e interesse por parte do usuário. 


\section{Considerações finais}

Desenvolvido por um grupo de alunos do curso Técnico em Informática para Internet na modalidade integrado ao ensino médio, esse projeto de observatório regional do trabalho e da educação profissional e tecnológica tem oportunizado: (i) integração entre os saberes desenvolvidos pelo discente ao longo de sua formação acadêmica com as demandas do mundo do trabalho; (ii) familiaridade do aluno de ensino médio com as dinâmicas da pesquisa, por permitir a esse visualizar o quanto seu saber, oriundo da área de informática, é capaz de gerar conhecimento ao conectar-se com outras áreas de formação; (iii) acesso a informações que podem orientar a definição de políticas públicas de expansão da educação profissional e tecnológica ofertadas na região, como também constituir as informações contidas no repositório como um instrumento imprescindível para a melhoria de cursos, programas e projetos de educação profissional e tecnológica ofertados pelo IFRS - Campus Rio Grande.

O avanço da tecnologia representa uma maior quantidade de ferramentas e aplicações, que visam facilitar o dia-a-dia de usuários, proporcionando um maior acesso a um conjunto de informações. A compilação de dados sob forma de um repositório significa, nesse trabalho, uma ferramenta de aprendizagem, pois permite ao aluno aplicar sua rede de saberes, problematizando seu campo de formação profissional. Sendo assim, a validade desse experimento torna-se altamente relevante para a educação técnica integrada ao ensino médio.

\section{Agradecimento}

Os autores gostariam de agradecer aos seguintes órgãos de fomento pelo financiamento do projeto o qual foi utilizado como estudo de caso para esse trabalho: Instituto Federal de Educação, Ciência e Tecnologia e o Conselho Nacional de Desenvolvimento Científico e Tecnológico (CNPq) pelas bolsas de auxílio ao estudante.

\section{Referências}

APL POLO NAVAL E OFFSHORE RIO GRANDE E ENTORNO. (2014) "Caracterização Territorial e Plano de Desenvolvimento.". PAES, R. (Org.). Rio Grande: Ed. da FURG.

APL POLO NAVAL E OFFSHORE/RS. (2015) "Arranjo Produtivo Local de Rio Grande/RS." Disponível em: <http://www.aplnavalriogrande.org>. Acesso em jul. de 2015.

BEHRENS, Marilda A.; MASETTO, Marcos T.; MORAN, José M. (2000) "Novas

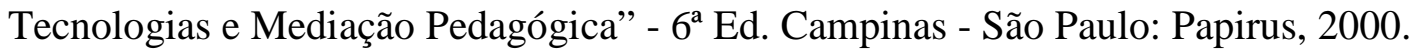

BELL, P.; BEER, B. (2015) "Introdução ao GitHub", São Paulo, SP. Disponível em: $<$ https://www.novatec.com.br/livros/introgithub/capitulo9788575224144.pdf>. Acesso em 18 ago. 2015.

CARVALHO, A. A.; SILVA, A.; SIMÕES, J. P. (2005) "Indicadores de qualidade de um site", Portugal, PT. Disponível em <http://repositorium.sdum.uminho.pt/bitstream/1822/7774/1/05AnaAmelia.pdf >. Acesso em 28 maio 2016. 
DEACON, J. (2013) "Model-View-Controller (MVC) Architecture". Disponível em <http://www.battersea-locksmith.co.uk/briefings/MVC.pdf> Acesso em 12 jun 2016.

IBICT, (2012). Instituto Brasileiro de Informação, ciência e Tecnologia. Disponível em $<$ http://dspace.ibict.br >. Acesso em agosto/2012.

IBGE. INSTITUTO BRASILEIRO DE GEOGRAFIA E ESTATÍSTICA. (2012) "Pesquisa Nacional por Amostra de Domicílios Contínua". Disponível em: <ftp://ftp.ibge.gov.br/Trabalho_e_Rendimento/Pesquisa_Nacional_por_Amostra_de_Do micilios_anual/2012/Sintese_Indicadores/comentarios2012.pdf>. Acesso out. 2015.

IBGE. INSTITUTO BRASILEIRO DE GEOGRAFIA E ESTATÍSTICA. (2014) "Pesquisa Nacional por Amostra de Domicílios Contínua". Disponível em: <ftp://ftp.ibge.gov.br/Trabalho_e_Rendimento/Pesquisa_Nacional_por_Amostra_de_Do micilios_continua/Notas_metodologicas/notas_metodologicas.pdf >. Acesso out. 2015.

ÍNDICE DE DESENVOLVIMENTO HUMANO MUNICIPAL BRASILEIRO.(2013) “Atlas do Desenvolvimento Humano no Brasil.” Brasília: PNUD, Ipea, FJP.

LEFEVRE, F.; LEFEVRE, A. M. C. (2005) "Depoimentos e discursos: uma proposta de análise em pesquisa social.” Brasília, DF: Liberlivro.

LEFEVRE, F.; LEFEVRE, A. M. C. (2010) "Pesquisa de Representações Sociais: um enfoque qualiquantitativo." Brasília, DF: Liberlivro.

LIBÂNEO, José Carlos.(2004) “Aprendizagem escolar e a formação de professores na perspectiva da pasicologia histórico-cultural e da teoria da atividade". Revista Educar, no 24, p. 113-147, Curitiba: Editora UFPR, 2004.

LIMA, A. L. D. I. "TIC na educação no Brasil: o acesso vem avançando. E a aprendizagem?” In: COMITÊ GESTOR DA INTERNET NO BRASIL. Pesquisa Sobre o Uso das Tecnologias de Informação e Comunicação no Brasil: TIC Educação 2011. São Paulo: CGI.br, 2012. Coord. Alexandre F. Barbosa. Trad. Karen Brito.

NETTO, C. M.; FABRI, L. B.W.; MORAES, H. N.; COSTA, A.C.R.; MORAES, R.C.R.; COSTA, H. R. (2012) "Repositórios institucionais de objetos de aprendizagem utilizando DSpace". In: Anais do $23^{\circ}$ Simpósio Brasileiro de Informática na Educação (SBIE 2012). Disponível em: <http://www.brie.org/pub/index.php/sbie/article/view/1767>. Acesso em: 10 maio 2016.

POLO NAVAL E OFFSHORE/RS. Arranjo Produtivo Local de Rio Grande/RS. Disponível em: <http://www.aplnavalriogrande.org>. Acesso em: 20 fev. de 2016.

RIBAMAR, F. S. (2006) "PostgreSQL Prático". Disponível em: <http://livros01.livrosgratis.com.br/ea000262.pdf>. Acesso em: 18 ago. 2015. 